\title{
Assessment of pork freshness based on changes in constituting chromophores using visible to near- infrared spectroscopy
}

Peyvasteh, M., Popov, A., Bykov, A., Meglinski, I.

M. Peyvasteh, A. Popov, A. Bykov, I. Meglinski, "Assessment of pork freshness based on changes in constituting chromophores using visible to near-infrared spectroscopy," Proc. SPIE 11202, Biophotonics Australasia 2019, 112020X (30 December 2019); doi: 10.1117/12.2539969

SPIE. Event: ANZCOP, 2019, Melbourne, Australia 


\title{
Assessment of pork freshness based on changes in constituting chromophores using visible to near-infrared spectroscopy
}

\author{
M. Peyvasteh ${ }^{1 *}$, A. Popov ${ }^{1}$, A. Bykov ${ }^{1}$, I. Meglinski $^{1,2,3}$ \\ ${ }^{1}$ Optoelectronics and Measurement Techniques, University of Oulu, Oulu, FI-90014, Finland \\ ${ }^{2}$ National Research Nuclear University "MEPhI", Institute of Engineering Physics for Biomedicine \\ (PhysBio), 115409 Moscow, Russia \\ ${ }^{3}$ Interdisciplinary Laboratory of Biophotonics, National Research Tomsk State University, 634050 \\ Tomsk, Russia
}

\begin{abstract}
Visible to near-infrared spectroscopy has been applied for non-invasive assessment of meat freshness. The measurements were done at room temperature with non-frozen pork samples. The absorbance spectra of the main chromophores in meat including oxymyoglobin, water, fat, and protein were different enough to be identified spectrophotometrically. The decreasing trend in absorbance spectra of these components over time can be associated with freshness decay. We used two configurations, fiber-optic probes and integrating sphere, to study their efficiency in meat quality evaluation. In the integrated sphere configuration, the samples experienced an immediate smooth decrease of oxymyoglobin absorbance arising from loss of superficial freshness, while degradation kinetics of water, fat and protein absorbance were detected after about 2.5 hours. In the fiber-optic configuration capable for sensing up to $570-\mu \mathrm{m}$ depth, the drop in oxymyoglobin absorbance started after 4.5 hours which would affect directly the color of sample associated with freshness.
\end{abstract}

Keywords: optical properties, pork freshness, vis-NIR spectroscopy, fiber optic, absorbance spectra, myoglobin

\section{INTRODUCTION}

Lipid oxidation, protein degradation and water loss are the consequences of freshness deterioration of meat during storage $^{1}$. Specifically, for pork, the quality of fresh product is traditionally classified into different categories based on color which is a determining factor for customers to evaluate freshness ${ }^{2,3}$. This is mainly referred to the amount of myoglobin types as a kind of protein existed in a packaged fresh meat, namely, Carboxymyoglobin, Oxymyoglobin, Deoxymyoglobin, and Metmyoglobin. Keeping the sample at room temperature, the forms of myoglobin interconvert and degrade through oxygenation, oxidation and reduction reactions, ultimately influencing the meat color which could be determining in freshness evaluation ${ }^{4}$. The majority of researches in this field have been done within external processes including storage or cooking while we studied freshness decay with non-frozen samples at room temperature.

\section{MATERIALS AND METHODS}

In this study, we applied visible- and near-infrared (NIR) light spectroscopy, which shows promise for fast and nondestructive analysis of biological materials. The pork steaks were purchased in a local supermarket and were kept at room temperature $\left(23^{\circ} \mathrm{C}\right)$ after removal from a fridge. In the first (table-top) configuration, an integrating sphere was used, which enabled acquisition of scattered light from the sample to properly account for absorption. In another configuration, we used a linear array of eleven optical fibers for sample illumination (1st fiber) and collection of light (11th fiber). The distance between the indicated fibers was $5.3 \mathrm{~mm}$; shorter distances are available using $2^{\text {nd }}-10^{\text {th }}$ fibers.

*motahareh.peyvasteh@oulu.fi

Biophotonics Australasia 2019, edited by Ewa M. Goldys, Brant C. Gibson, Proc. of SPIE Vol. 11202, 112020X · C 2019 SPIE · CCC code: 0277-786X/19/\$21 · doi: 10.1117/12.2539969 


\section{RESULTS}

Absorbance changes over time for specific wavelengths attributed to the presence of meat chromophores are presented in Figure 1, for the integrating sphere configuration in the visible (a) and NIR (b) spectral ranges and for the fiber-optic configuration in the visible spectral range (c). It has been observed that for the both visible and NIR spectral regions, integrated absorbance for different chromophores experienced a decreasing trend, which could affect pork freshness.

(a)

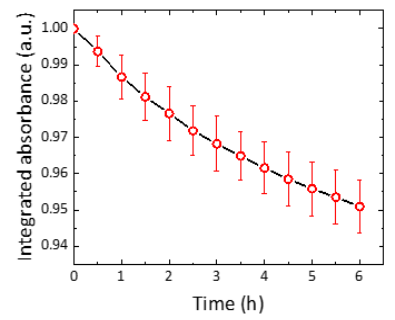

(b)

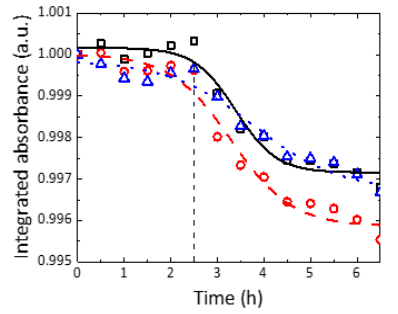

(c)

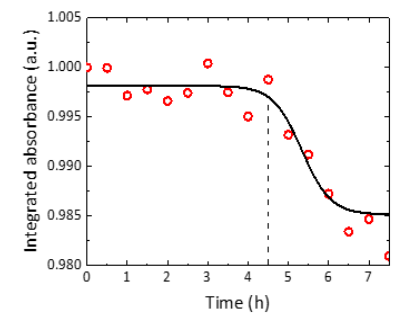

Figure 1. Degradation kinetics of (a) oxymyoglobin ${ }^{2}$, (b) fat ${ }^{2}\left(1200 \mathrm{~nm}\right.$, triangle/dot), water ${ }^{5}$ (1450 nm, square/solid) and proteins ${ }^{6}(1520$ and $1600 \mathrm{~nm}$, circle/dash) measured in the integrating sphere configuration and (c) oxymyoglobin measured in the fiber-optic configuration. The dashed vertical line in (b) and (c) indicates the transition time between the fresh and spoiled state of the studied pork sample.

Specifically, degradation of oxymyoglobin indicating color changes started from the beginning in the integrating sphere setup (Fig. 1a), while in the NIR region (Fig. 1b), integrated absorbance decreased slower starting from approximately 2.5 hours. The difference between the two configurations is in the probing depth, which was shallower in the integrating sphere setup due to higher contribution of the surface and subsurface reflected photons. In the fiber-optic configuration, the probing depth was managed through changing the source-detector separation, i.e. by choosing the proper detecting fiber (since the illuminating fiber was the same).

\section{CONCLUSIONS}

According to the results, it is possible to observe the decreasing absorbance trend for different pork chromophores in both visible and NIR spectral ranges showing loss of freshness over time. In the visible range, absorbance changes at 540- and 580-nm wavelengths allow for monitoring degradation of oxymyoglobin ${ }^{7}$ associated with freshness loss. In the NIR region, absorbance changes of fat $(1200 \mathrm{~nm})$, water $(1450 \mathrm{~nm})$, and proteins $(1525$ and $1600 \mathrm{~nm})$ show promise to detect spoilage. Specifically, absorbance of the superficially located oxymyoglobin decays immediately but only after 4.5 hours at the depth of $0.57 \mathrm{~mm}$, while absorbance of surface water and protein components experiences a steep decrease only after about 2.5 hours associated with larger penetration depth of NIR light compared to visible wavelength.

\section{REFERENCES}

[1] Dave, D. and Ghaly, A., "Meat spoilage mechanisms and preservation techniques: A critical review," American Journal of Agricultural and Biological Sciences, 6(4), 486-510 (2011).

[2] ElMasry, G. and Nakauchi, S. "Prediction of meat spectral patterns based on optical properties and concentrations of the major constituents," Food Sci. Nutr. 4(2), 269-283 (2016).

[3] Alander, J., Bochko, V., Martinkauppi, B., Saranwong, S., and Mantere, T., "A review of optical nondestructive visual and near-infrared methods for food quality and safety," International Journal of Spectroscopy, 341402 (2013).

[4] Mancini, R. A. and Hunt, M., "Current research in meat color," Meat Science, 71(1), 100-121 (2005).

[5] Firtha, F., Jasper, A., and Friedrich, L. "Spectral and hyperspectral inspection of beef ageing state," Presented at Chinese-European cooperation for a long-term sustainability. Budapest, Hungary (2011).

[6] Barbin, D., Kaminishikawahara, C., and Soares, A., "Prediction of chicken quality attributes by near infrared spectroscopy," Food Chem. 168, 554-580 (2015).

[7] Park, B., Chen, Y., Hruschka, W., and Koohmaraie, M. "Principal component regression of NIR reflectance spectra for beef tenderness prediction" ASABE. 44(3), 609-615 (2001) 